\title{
DIFFRACTIVE DIS CROSS SECTIONS AND PARTON DISTRIBUTIONS
}

\author{
F.-P. SCHILLING (H1 COLLABORATION) \\ Physics Department, CERN, CH-1211 Geneva 23, Switzerland \\ E-mail: frank-peter.schilling@cern.ch
}

\begin{abstract}
Highlights are presented mainly from two recent measurements of the diffractive Deep Inelastic Scattering cross section at HERA. In the first, the process $e p \rightarrow e X p$ is studied by tagging the leading final state proton. In the second, events of this type are selected by requiring a large rapidity gap devoid of hadronic activity in the proton direction. The two measurements are compared in detail and the kinematic dependences are interpreted within the framework of a factorisable diffractive exchange. Diffractive parton distributions are determined from a next-toleading order QCD analysis of the large rapidity gap data, which can be applied to the prediction of diffractive processes, also at the TEVATRON and the LHC.
\end{abstract}

\section{Introduction}

This report ${ }^{\mathrm{a}}$ summarises recent results on measurements of the diffractive deepinelastic scattering (DIS) cross section obtained with the $\mathrm{H} 1$ detector at the HERA $e p$ collider, in particular from two recent publications ${ }^{1,2}$. The measurements cover an unprecedented kinematic range of photon virtualities $3.5<Q^{2}<1600 \mathrm{GeV}^{2}$ with unprecedented precision (5\% statistical, $5 \%$ systematic and $6 \%$ normalisation errors in the best-measured region).

In the first paper ${ }^{1}$, the Forward Proton Spectrometer (FPS) is used to detect and measure the four-momentum of the outgoing proton in the process $e p \rightarrow e X p$. This selection method has the advantages that the proton unambiguously scatters elastically and that the squared four-momentum transfer at the proton vertex $t$ can be reconstructed. However, the available statistics are limited by the FPS acceptance. A high statistics sample of diffractive DIS events is selected on the basis of a large rapidity gap (LRG) in the outgoing proton direction, as described in the second paper ${ }^{2}$. The measured process is $e p \rightarrow e X Y$ where $Y$ corresponds to any baryonic state with mass $M_{Y}<1.6 \mathrm{GeV}$.

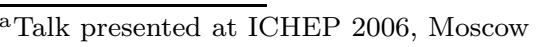

Together, the FPS and LRG data provide a means of studying inclusive diffraction as a function of all relevant variables. In addition to $t$ and the usual DIS variables $x$ and $Q^{2}$, measurements are made as a function of the fractional proton longitudinal momentum loss $x_{\mathbb{P}}$ and of $\beta=x / x_{\mathbb{P}}$, which corresponds to the fraction of the exchanged longitudinal momentum carried by the quark coupling to the virtual photon.

The data exhibit a remarkable consistency with proton vertex factorisation ${ }^{3}$, where the dependences on $x_{\mathbb{P}}, t$ and $M_{Y}$ describing the proton vertex are completely independent of $\beta$ and $Q^{2}$, which describe the hard interaction with the photon. The dependences on $x_{\mathbb{P}}$ and $t$ can then be expressed in terms of an effective pomeron flux of colourless exchange, whilst the $\beta$ and $Q^{2}$ dependences can be interpreted in terms of diffractive parton distributions (DPDFs), which describe the partonic structure of that exchange ${ }^{4}$.

Only a short summary of a few highlights is possible here. Much more detail, including the multi-differential cross section measurements themselves, can be found $\mathrm{in}^{1,2}$. The first charged current diffractive measurement, as well as ratios of the diffractive and inclusive cross sections, are also presented 

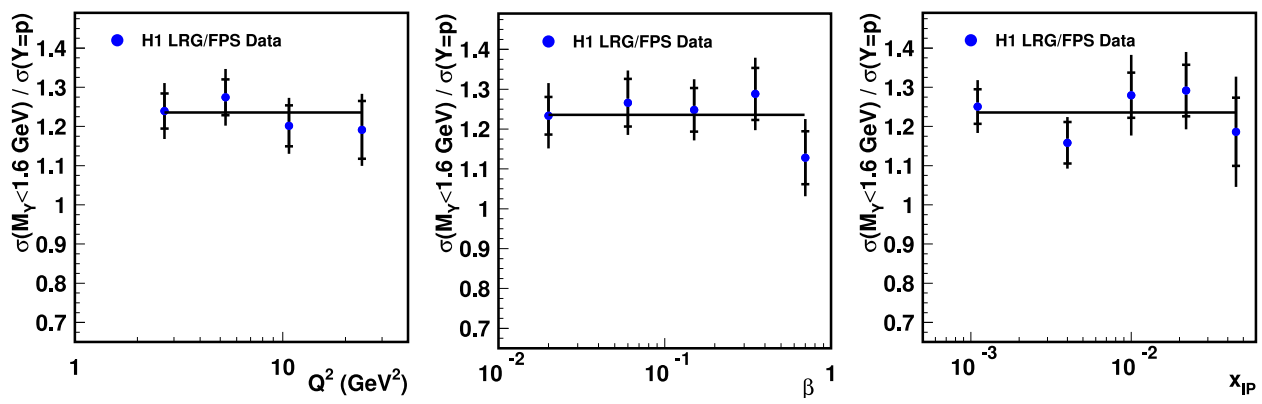

Fig. 1. The ratio of the cross section for $M_{Y}<1.6 \mathrm{GeV}$ and $|t|<1 \mathrm{GeV}^{2}$ (LRG data) to that for $Y=p$ and $|t|<1 \mathrm{GeV}^{2}$ (FPS data) as a function of $Q^{2}, \beta$ and $x_{\mathbb{P}}$, averaged over the other variables. A $13 \%$ normalisation uncertainty is not shown.

in $^{2}$, but not covered here. New H1 diffractive DIS measurements with increased statistical precision, but in a limited kinematic range, were also presented ${ }^{5}$.

\section{Comparison between Data Sets}

Since the LRG and FPS data sets are statistically independent and have very different systematics, the two measurements constitute a powerful mutual cross-check. Compatibility between them is established in detail by performing $t$-integrated measurements by both techniques with identical binning and forming the ratio of the two measurements for each $\left(Q^{2}, \beta, x_{\mathbb{P}}\right)$ point $^{1}$.

The dependences of this ratio on each kinematic variable individually is shown in Fig. 1 after taking statistically weighted averages over the other two variables. Within the uncertainties of typically $10 \%$ per data point, there is no significant dependence on $\beta, Q^{2}$ or $x_{\mathbb{P}}$. The ratio of overall normalisations, LRG / FPS, is $\sigma\left(M_{Y}<1.6 \mathrm{GeV}\right) / \sigma(Y=$ $p)=1.23 \pm 0.03$ (stat.) \pm 0.16 (syst.), consistent with predictions for the proton-elastic cross section and the proton dissociation cross section with $M_{Y}<1.6 \mathrm{GeV}^{1}$. The FPS data are also consistent with the corresponding measurement obtained with the ZEUS Leading Proton Spectrometer ${ }^{6}$.

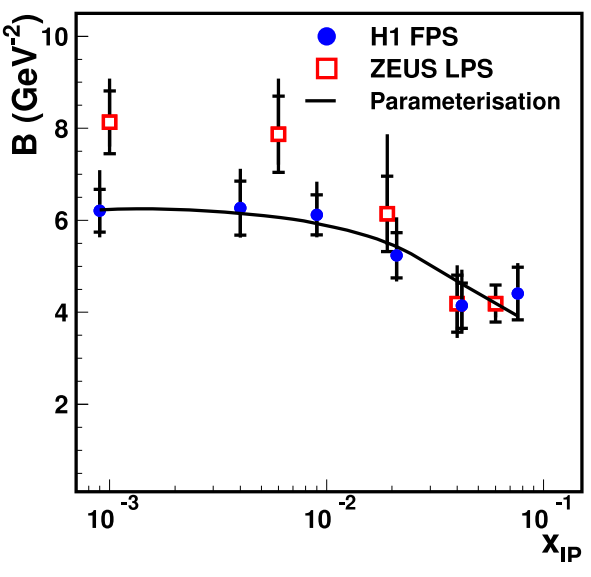

Fig. 2. Measurements of the slope parameter $B$ by $\mathrm{H} 1$ and ZEUS and a parameterisation of the $\mathrm{H} 1$ data as used to describe the pomeron flux factor.

\section{Dependences on $x_{\mathbb{P}}$ and $t$}

The $t$ dependences of diffractive cross sections are commonly parameterised with an exponential, $d \sigma / d t \sim e^{B t}$. The values of $B$ resulting from such fits to the FPS data are shown as a function of $x_{\mathbb{P}}$ in Fig. 2. At low $x_{\mathbb{P}}$, the data are compatible with a constant slope parameter, $B \simeq 6 \mathrm{GeV}^{-2}$. In a Regge approach with a single linear exchanged pomeron trajectory, $\alpha_{\mathbb{P}}(t)=\alpha_{\mathbb{P}}(0)+\alpha_{\mathbb{P}}^{\prime} t$, the slope parameter decreases with increasing $x_{\mathbb{P}}$ according to $B=B_{0}-2 \alpha_{\mathbb{P}}^{\prime} \ln x_{\mathbb{P}}$. The low $x_{\mathbb{P}}$ data thus favour a small value of $\alpha_{\mathbb{P}}^{\prime} \simeq 0.06 \mathrm{GeV}^{-2}$, though $\alpha_{\mathbb{P}}^{\prime} \simeq 0.25$, as obtained from soft hadronic interactions, 
cannot be excluded.

The $x_{\mathbb{P}}$ dependences of both measurements are interpreted in terms of effective pomeron intercepts. The two results are consistent, the more precise value of $\alpha_{\mathbb{P}}(0)=1.118 \pm 0.008$ (exp. $)_{-0.010}^{+0.029}$ (model) coming from the LRG data. The dominant error arises from the strong positive correlation between $\alpha_{\mathbb{P}}(0)$ and $\alpha_{\mathbb{P}}^{\prime}$, such that $\alpha_{\mathbb{P}}(0)$ increases to around 1.15 if $\alpha_{\mathbb{P}}^{\prime}$ is set to $0.25 \mathrm{GeV}^{-2}$ rather than $0.06 \mathrm{GeV}^{-2}$. The extracted $\alpha_{\mathbb{P}}(0)$ is slightly higher than the 'soft pomeron' value of $\alpha_{\mathbb{P}}(0) \simeq 1.08$, obtained from long distance hadronic interactions. The values of both $\alpha_{\mathbb{P}}(0)$ and $\alpha_{\mathbb{P}}^{\prime}$ describing diffractive DIS are compatible with the results obtained for soft exclusive photoproduction of $\rho^{0}$ mesons $^{7}$. This similarity supports the picture of diffractive DIS as probing the structure of a 'soft' pomeron. 'Hard' perturbative two gluon exchange contributions are likely to be small, as is also suggested by the lack of a signal for exclusive dijet production ${ }^{8}$.

Further analysis in which either the slope $B$ or the intercept $\alpha_{\mathbb{P}}(0)$ is allowed to vary with $\beta$ or $Q^{2}$ shows no significant dependences (Fig. 3), confirming the validity of proton vertex factorisation for the present data. This contrasts with the $Q^{2}$ dependent effective pomeron intercept extracted in a Regge approach to inclusive low $x$ proton structure function data, as studied in detail via the ratio of diffractive to inclusive cross sections $\mathrm{in}^{2}$.

\section{Dependences on $\beta$ and $Q^{2}$ : Diffractive Parton Densities}

$\mathrm{In}^{2}$, the cross section is presented differentially in $\beta, Q^{2}$ and $x_{\mathbb{P}}$. After dividing out the $x_{\mathbb{P}}$ dependence using a flux factor with parameters obtained as described in section 3 , the results from different $x_{\mathbb{P}}$ values are compatible, as expected where proton vertex factorisation holds.

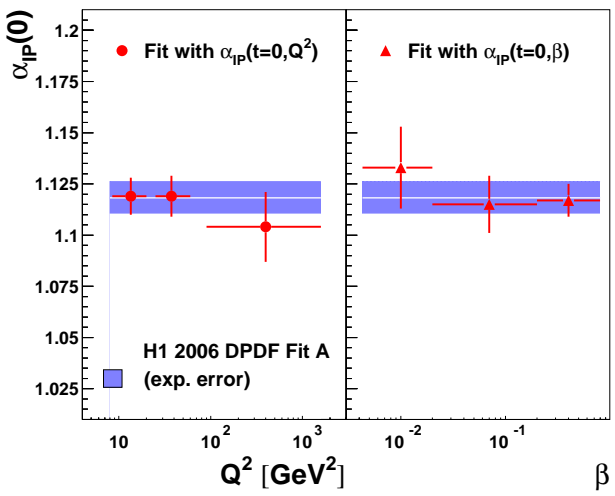

Fig. 3. Effective pomeron intercept $\alpha_{\mathbb{P}}(0)$ as extracted from the LRG data, showing no significant variation with $Q^{2}$ or $\beta$.

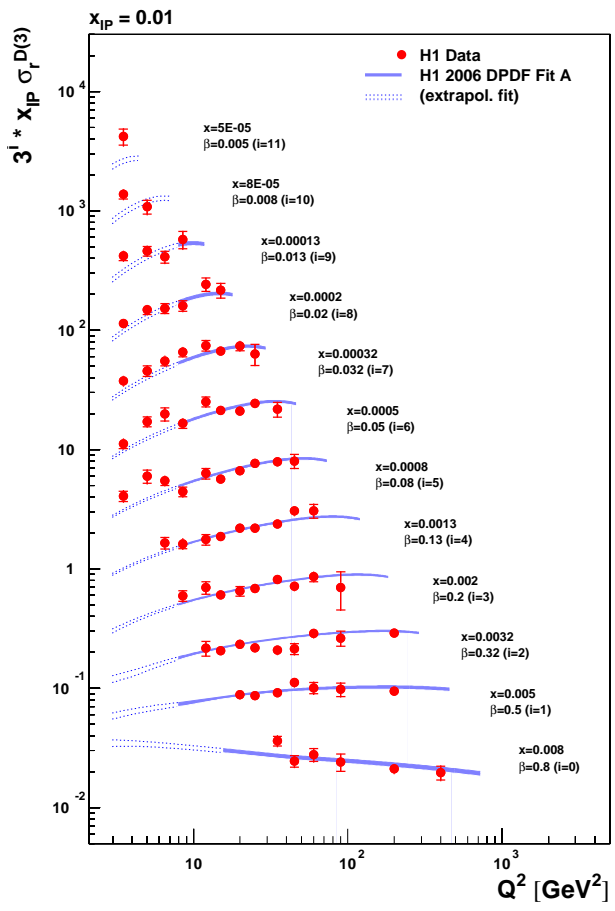

Fig. 4. $Q^{2}$ dependence of the diffractive DIS cross section at $x_{\mathbb{P}}=0.01$ for different values of $\beta$.

The $\beta$ and $Q^{2}$ dependences (Fig. 4) of the data are interpreted in a next-to leading order (NLO) DGLAP QCD fit ${ }^{2}$ in order to extract DPDFs. For the first time, experimental and theoretical uncertainties are evaluated for these partons. The results are shown in Fig. 5. 


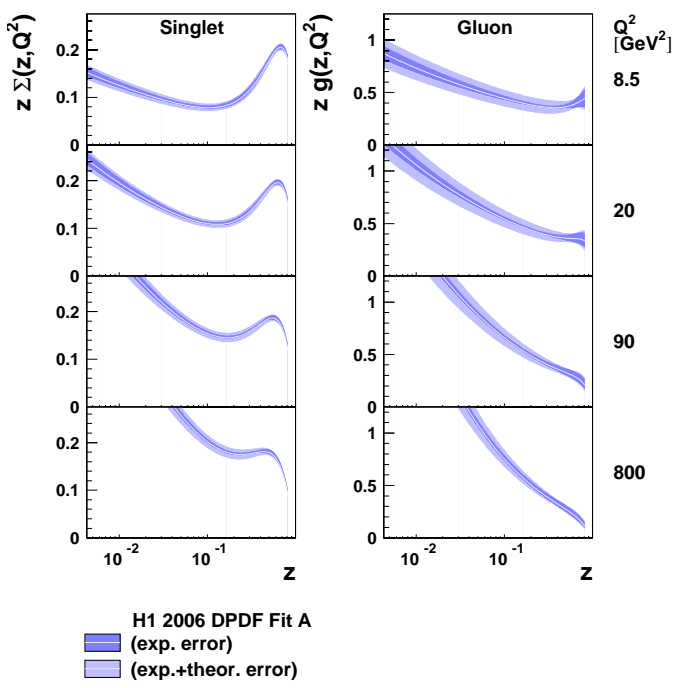

Fig. 5. Quark singlet and gluon distributions from the NLO QCD fit 'H1 2006 DPDF Fit A', as a function of the momentum fraction $z$ carried by the relevant parton.

The singlet quark density is very closely related to the measured diffractive cross section and is thus well constrained, with a typical error of $5 \%$. According to the DGLAP evolution equations, the logarithmic $Q^{2}$ derivative (shown in Fig. 6 for $x_{\mathbb{P}}=0.01$ ) contains contributions due to the splittings $g \rightarrow q \bar{q}$ and $q \rightarrow q g$, convoluted with the diffractive gluon and quark densities, respectively. The derivative is determined almost entirely by the diffractive gluon density up to $\beta \simeq 0.3$. The large positive $\ln Q^{2}$ derivatives in this region can thus be attributed to a large gluonic component in the DPDFs. For $\beta>0.3$, the contribution to the $Q^{2}$ evolution from quark splittings $q \rightarrow q g$ becomes increasingly important and the derivatives become less sensitive to the gluon density. The gluon density is thus known to around $15 \%$ at low $\beta$, with an uncertainty that grows quickly for $\beta>0.3$.

These DPDFs provide important input to final state measurements such as those involving jets and charm quarks ${ }^{9}$, which may also provide important additional constraints $^{10}$ on the gluon at high $\beta$. In-

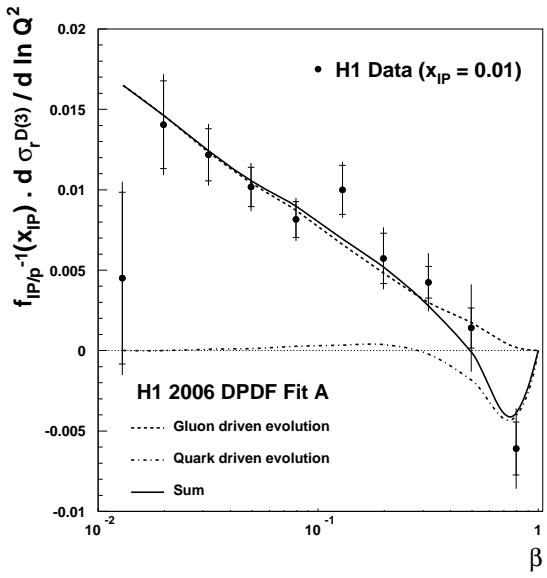

Fig. 6. Logarithmic $Q^{2}$ derivatives of the diffractive cross section at fixed $x_{\mathbb{P}}=0.01$ as a function of $\beta$.

tegrated over $\beta$, the gluon density carries around $70 \%$ of the total momentum. A similar fraction of the total proton momentum is carried by the inclusive gluon density in the low $x$ region where valence quark effects are small. This similarity of the ratio of quarks to gluons in the DPDFs and the inclusive proton parton densities is reflected ${ }^{2}$ in a ratio of the two cross sections which, to good approximation, is flat as a function of $Q^{2}$ at fixed $x$ and $x_{\mathbb{P}}$.

The DPDFs may also be used in calculations of diffractive cross sections at the TEVATRON as well as the LHC.

\section{References}

1. H1 Coll., DESY06-048, hep-ex/0606003, acc. by Eur. Phys. J. C.

2. H1 Coll., DESY06-049, hep-ex/0606004, acc. by Eur. Phys. J. C.

3. G. Ingelman and P. Schlein, Phys. Lett. $\boldsymbol{B}$ 152, 256 (1985).

4. J. Collins, Phys. Rev. D 57, 3051 (1998) [Erratum-ibid. D61, 019902 (2000)].

5. H1 Coll., H1prelim-06-014.

6. ZEUS Coll., Eur. Phys. J. C 38, 43 (2004).

7. K. Krueger, these proceedings.

8. H1 Coll., Eur. Phys. J. C 20, 29 (2001)

9. M. Kapishin, these proceedings.

10. H1 Coll., H1prelim-06-016. 